\title{
Visual Analysis of Research on Precise Funding Fine Chemical in Universities Based on Knowledge Mapping
}

\author{
Qing Xu, Xiaolan Wang, Wenhao Ying* \\ Changshu Institute of Technology, Changshu, Jiangsu, 215500, China \\ Corresponding author.
}

\begin{abstract}
The article utilizes Citespace software to analyze the existing research literature, uses the method of word frequency analysis to conduct content mining and data processing on the journal literature in the field of precision funding fine chemical industry research in colleges and universities, and analyzes the history and research of the precision funding of colleges and universities for students with financial difficulties in recent years. The current situation is expected to provide reference and reference for follow-up researchers, and to provide ideas and suggestions for practically improving the level of university funding.
\end{abstract}

Keywords: Fine chemical research; precision funding for colleges and universities; visual analysis; citespace

\section{Introduction}

The issue of financial aid for students from colleges and universities with financial difficulties is related to the realization of educational equity, the overall victory of the country's fight against poverty and the ultimate realization of a comprehensively well-off society. In 2016, the Ministry of Education, the National Development and Reform Commission, the Ministry of Civil Affairs, the Ministry of Finance, the Ministry of Human Resources and Social Security, and the National Office of Poverty Alleviation jointly issued the "Thirteenth Five-Year Plan for Poverty Alleviation through Education". Since then, the country has launched a comprehensive fight against poverty in education. The prologue. Educational poverty alleviation is an important part of the country's fight against poverty through education, and it is a political task with a great responsibility. In recent years, the state has provided more and more financial aid to college students from poor families. At present, colleges and universities have basically achieved the goal of not letting a poor student drop out of school. However, there are still groups of college students with financial difficulties in their families. How to improve the funding system of awards, loans, diligence, compensation and reduction, implement accurate funding, provide necessary and stable economic security, and help students with financial difficulties to complete their studies are the focus of the society and colleges[1]. In the overall layout of the national "Thirteenth Five-Year Plan" for student funding, the concept of "precise funding" has been implemented throughout. Therefore, "precision funding" has become a new focus of college funding. At present, domestic scholars have done a lot of theoretical research in this area, and have accumulated a lot of research results so far. This article aims to use the method of content analysis, using Citespace software, to document the research results accurately funded by colleges and universities from 2015 to 2020 . Quantitative analysis, reviewing and analyzing the history and current situation of my country's colleges and universities' precision funding to students with financial difficulties in recent years, in order to provide reference and reference for follow-up researchers, and provide ideas and suggestions for effectively improving the level of college funding.

\section{Research Method}

\subsection{Data sources}

By using the method of content analysis, this paper conducts a comprehensive search in China Full-text Journal Database of CNKI, with the key word of "precision funding of colleges and universities" on May 29, 2020. A total ISSN: 0010-8189 
of 658 records were obtained from 2015 to 2020, excluding newspaper records, and finally 656 literature records were obtained. The exported data includes literature type, author, journal, keywords, and so on Abstract, organization and other information [2]. Select "refworks" as the export file format, and name the export document "download_XXXX". At present, CNKI supports up to 500 records to be exported at one time, so the export process needs to be repeated.

\subsection{Application software}

CiteSpace visualization software is used for data analysis software, which is developed by Chaomei Chen, Professor of Drexel University and professor of Yangtze River scholars at Dalian University of technology [3]. From the author cooperation network, research institutions cooperation network, terminology, keywords, sources and other dimensions, using the word frequency analysis method, through the frequency detection and change analysis of the subject words and keywords, to determine the research hotspot, frontier and future development trend of University precision funding.

\section{Literature Research and Analysis of Precision Funding in Colleges and Universities in China}

\subsection{Annual trend of the literature}

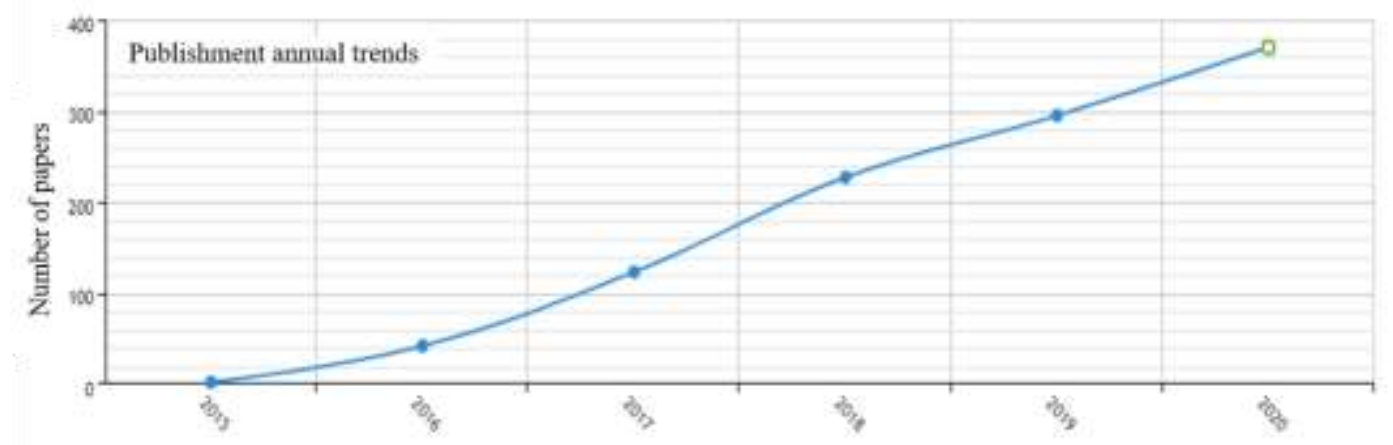

Fig.1. Annual trend chart of the number of precise funding documents in universities

To a certain extent, the number of domestic scholars' papers on precise financial aid for students from poor families reflects the theoretical level and activity of academic research in this field. The more the number of literatures, the more attention researchers pay to this field [4]. Before 2015, the number of research literature on precise funding for students from poor families in Colleges and universities in China was small, which began to increase year by year in 2015, and basically maintained a good upward trend from 2015 to 2020, and the number of research results increased year by year (shown as Figure 1). From the overall point of view, the distribution of the number of literature is in line with the development of precise funding research for students from poor families in Colleges and universities in China.

\subsection{Analysis of author collaboration network}

At present, CiteSpace software can only process documents exported in wos format, and CNKI source data can only be used for software analysis after data transformation. In CiteSpace 5.6.R5, the preprocessed data source is imported, the time zone is 2015-2020, the time span is 1 year, the network node selects the author, the threshold (C, $\mathrm{C}, \mathrm{CCV})$ is set to $(2,2,5)(2,2,5)(2,2,5)$, and the data extraction object is Top50.

The software running results show that, as shown in Figure 2, within the threshold range, 43 nodes are generated, 12 lines are connected, and the nodes are concentrated in 2017-2019. Qu Lili Li, Hou Yunchen, Zhang Jingke and Jiang Junyi all appeared three times, while the other 39 authors all appeared two times. There are three links among

ISSN: 0010-8189 
Qu Lili Li, Peng Weibin and Chai Zheng, and they form a cluster; There are three links among Zhang Jingke, Xie Haoran and Jiang Junyi; In addition, there are connections between Liu Ming and Xu Zhongyuan, He Jing and Lei Wen, Zhang Jianrong and Jin Xiaoping, pan Dong and Feng Jiao, and Zhang Ke and Wen Zhanming. It can be seen from the results that the correlation between the authors in the research field of efficient and accurate funding is low, and they are basically in the situation of "fighting on their own". At most, there are small teams within the organization.

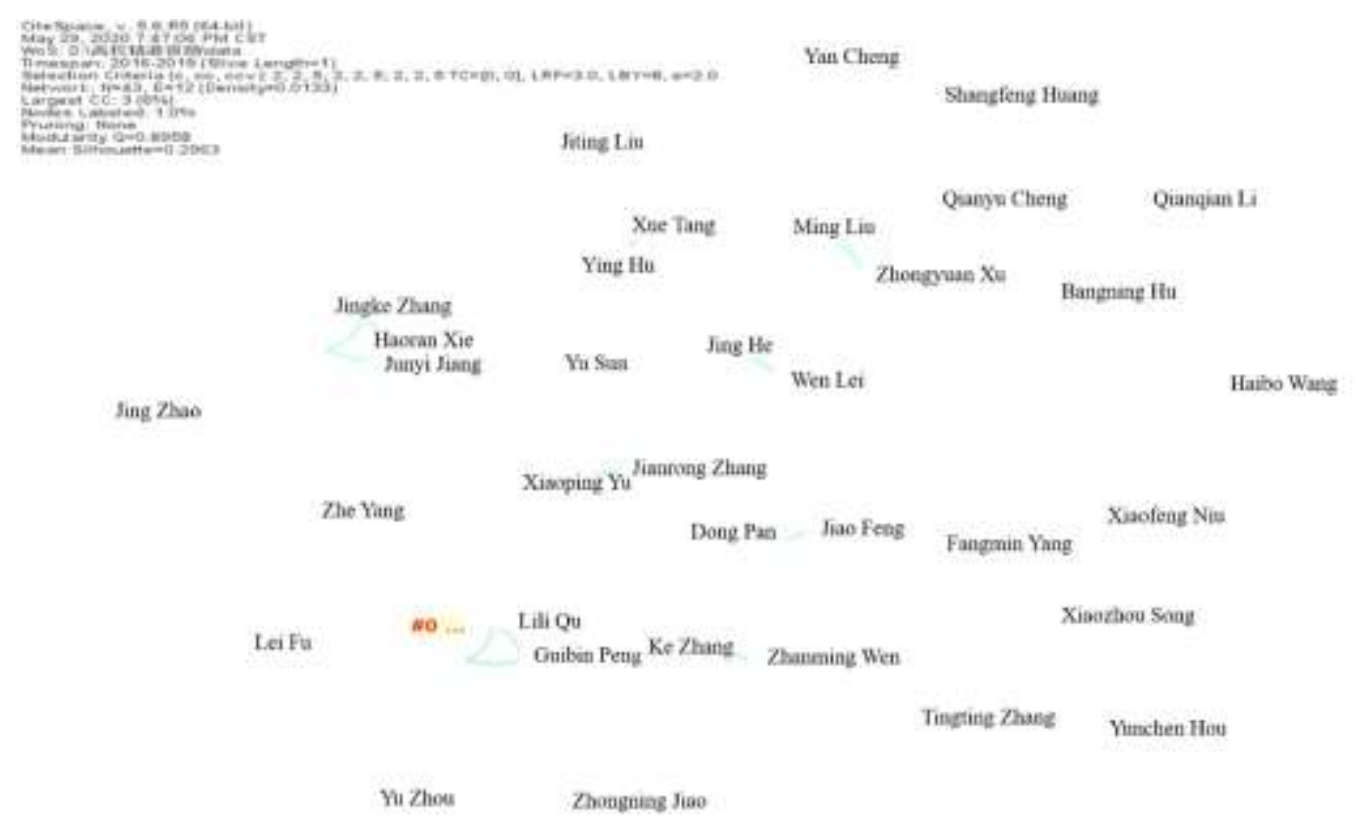

Fig.2. A network map of author cooperation funded by colleges and universities

3.3 Institutional cooperation network analysis

When the target data is imported into Citespace 5.6.R5, the network node selection organization "institution", other settings are the same as the previous article, the software running results show [5], within the threshold range, 37 nodes are generated, and the connection is 0 . The frequency of occurrence is shown in Table 1. The results of software operation show that there is no connection between the institutions, which indicates that there is no institutional cooperation in the field of precision funding research in Colleges and universities, and the core group of institutions has not been formed. All of them are internal student work lines or ideological and political researchers to carry out their own research. The number of papers published by more than 200 institutions is only one, which indicates that most institutions lack sustained attention to the research in this field. In addition, the emergence years of high-frequency institutions are mainly concentrated in 2018 and 2019, which is related to the implementation time of targeted poverty alleviation policy in Colleges and universities after the implementation of the national poverty alleviation policy.

Table 1 Cooperative network map of research institutions in the field of precision funding of universities

\begin{tabular}{ccc}
\hline Research institute & Year & Frequency of occurrence \\
\hline Xuzhou Medical University & 2019 & 6 \\
Jiangsu Business Vocational School & 2018 & 4 \\
Wuxi Institute of Arts and Technology & 2019 & 4 \\
Nanjing University of Posts and Telecommunications & 2018 & 3
\end{tabular}

ISSN: 0010-8189

C CONVERTER 2020 


\section{Research Hotspot of Precision Funding in Colleges and Universities Based on Knowledge Mapping}

Research hotspots refer to the scientific issues discussed by a large number of related literatures in a certain period of time. Although there are only 3-5 keywords in the literature, they are the core and essence of the whole literature. They are highly summarized and condensed literature topics. Therefore, when analyzing literature keywords, high-frequency keywords are often used to identify as hot issues in a certain research field [6].

Import the target data into citespace5.6.R5, select the term "Term", keyword "Keyword", and source "Source" for the network node, and set the threshold $(\mathrm{C}, \mathrm{C}, \mathrm{CCV})$ to $(2,2,10)(2,2), 10)(2,2,10)$, the data extraction object is top50. The software running result shows that 114 nodes are generated and 415 connections are made. High-frequency words are shown in Table 2.

The software running results show that, as shown in Figure 3, there are seven large clusters, the two largest clusters (\# 0) (\# 1) have 18 members, the silhouette value of (\# 0 ) is 0.759 , and the LLR label is "new exploration of University funded education work under the background of targeted poverty alleviation", The most active one in this cluster is the "never let a child drop out of school because of poverty" published by Yin Xiaojun (2017) in Gansu Education\# 1) The silhouette value is 0.659, and the LLR label is "Research on the contradiction between supply and demand of university funding in the new era -- a new conclusion based on the transformation of major social contradictions", among which the most active one is "dynamic priority management mechanism for accurate identification of university funding objects" published by Yin Xiujuan (2018) in Yuejiang University Journal. Through the study of inflation words, it is found that the first mutation rate is precision funding, with a mutation rate of 2.42 , followed by funding management, with a mutation rate of 1.94 , indicating that precision funding and funding management is the future development trend of college student funding.

It can be seen from Table 2 that in addition to precision funding, funding education, precision poverty alleviation, big data, students from poor families, path, education equity are also hot spots in the research field of precision funding in Colleges and universities. Among them, the intermediary centrality of students with financial difficulties is 0.22 , which indicates that students with financial difficulties are the core objects in the research field of precision funding in Colleges and universities. The intermediary centrality of targeted poverty alleviation and subsidized education reached 0.20 and 0.19 respectively, indicating that subsidized education and targeted poverty alleviation play a very important role in the research field of targeted funding in Colleges and universities.

Table 2 High-frequency keywords in the literature of "precision funding for universities" (top 20)

\begin{tabular}{ccc}
\hline High-frequency keywords & Frequency & Betweenness centrality \\
\hline precision funding & 322 & 0.31 \\
colleges and universities & 141 & 0.20 \\
subsidizing education & 126 & 0.19 \\
targeted poverty alleviation & 116 & 0.20 \\
big data & 75 & 0.12 \\
poor students & 52 & 0.14
\end{tabular}

ISSN: 0010-8189

(C) CONVERTER 2020 
poor college students

students from poor families

student funding

funding

supporting work

university funding

college students

path

educating people

university student

new era

education equity

precision
42

38

26

25

22

19

19

18

18

17

15

15

14

13
0.12

0.22

0.12

0.10

0.12

0.10

0.07

0.07

0.06

0.08

0.09

0.04

0.05

0.01
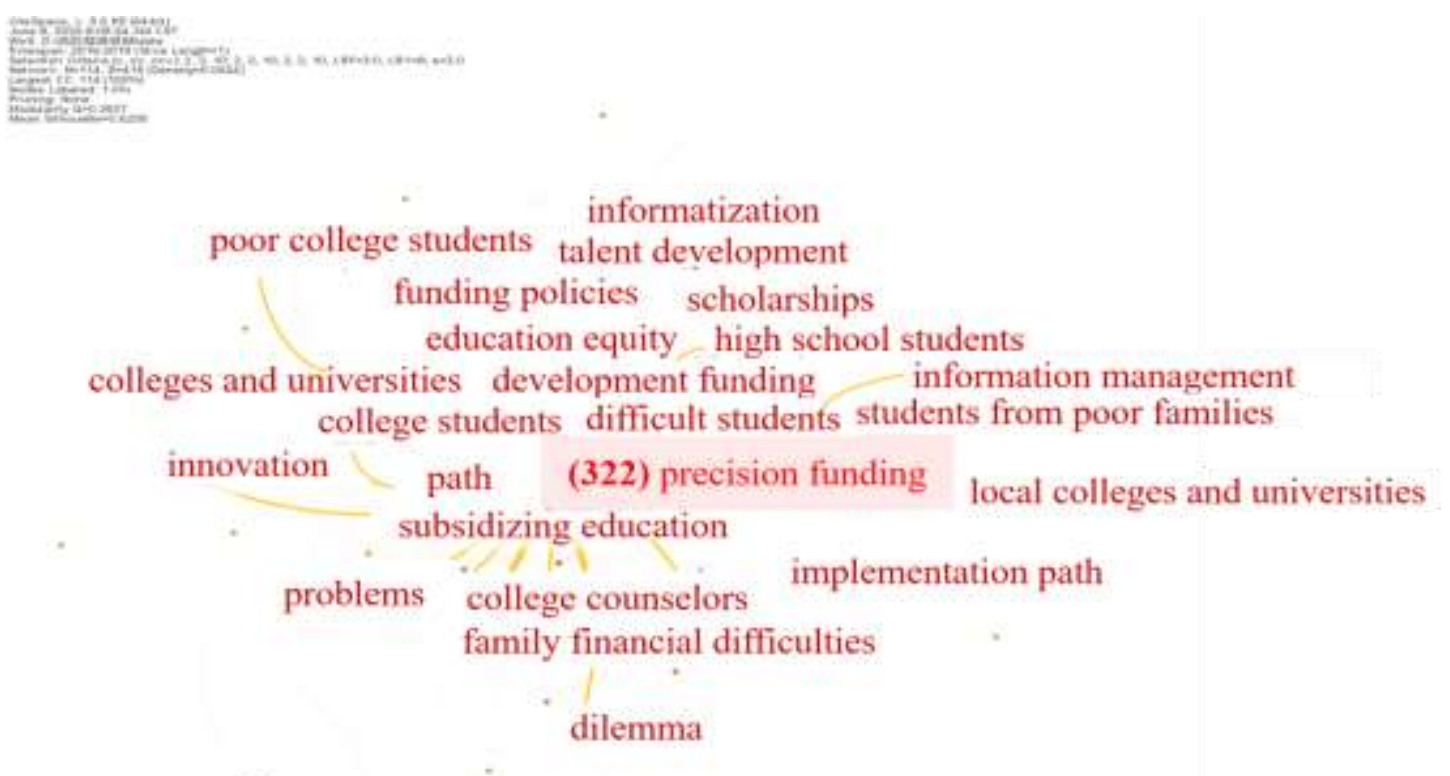

Fig.3. Knowledge mapping of hotspots for precision funding research in universities

\section{Knowledge Evolution of Precision Funding Research in Chinese Universities}

In order to better show the research hotspots in the field of precision funding in colleges and universities in China, the time axis is set to 2015-2020, the time interval is one year, the network node selects "keyword", other settings are the same as above, and the time zone knowledge map of key words in precision funding research in colleges and universities in China is generated (shown as Figure 4). According to the emergence of time zone keywords, it can directly reflect the evolution trend of research hotspots in this field. As shown in Figure 3, the research on precision funding of colleges and universities in China has gone through a process from simple to deep. From the early embryonic stage, the hot keywords are mainly "college students, precision funding, funding policies, models, university funding work" and so on; 2017-2018 is a period of rapid development, and the hot keywords are mainly ISSN: 0010-8189 
"funding system, accurate identification, education equity, new media, information management, funding education, accurate assistance" and so on; 2019 is a breakthrough development stage, and the hot keywords are "funding education system, Sanquan education, talent development, funding education mode, innovation" and so on.

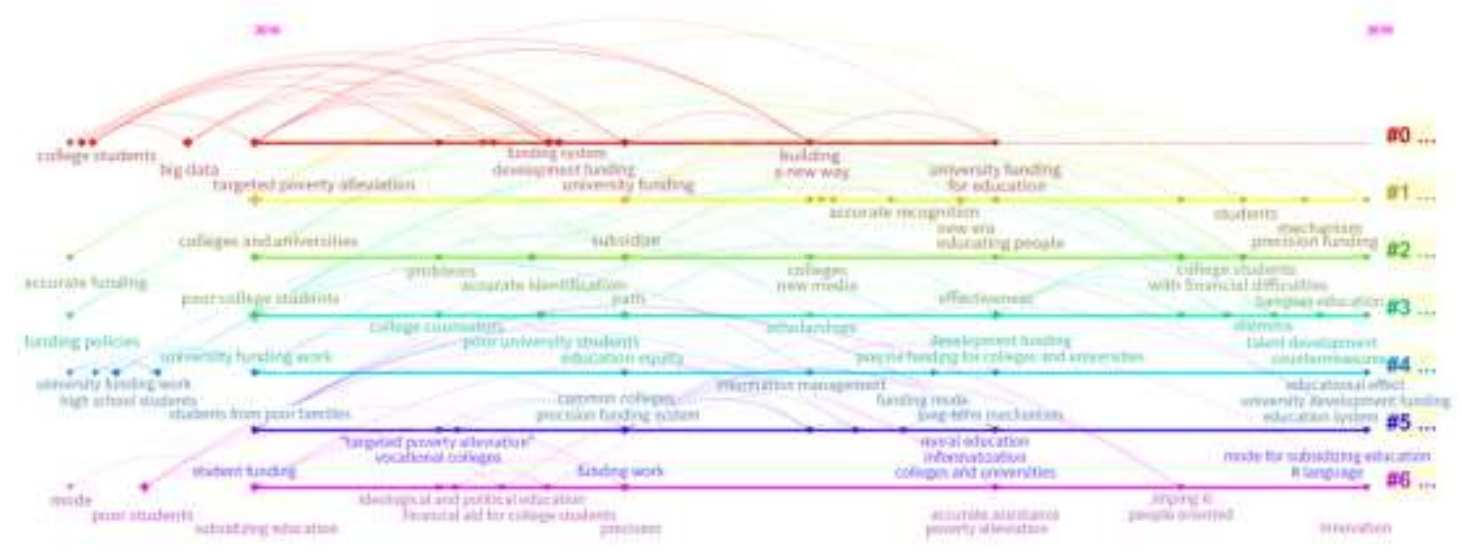

Fig.4. Keywords time zone knowledge mapping for precise funding of universities

\section{Conclusion and Suggestion}

6.1 Research institutions are scattered, and no core author group has been formed.

Since 2013, when general secretary Xi Jinping visited Xiangxi, Hunan, the concept of "precision poverty alleviation" was put forward for the first time [7], the idea of "precise funding" runs through the overall layout of student financial assistance in the 13th Five-Year plan. Universities and governments gradually began to pay attention to the research of precision funding in colleges and universities, and published some research results. In 2018 and 2019, the number of research shows explosive growth and remains at a high level. From the perspective of research institutions, there are few institutional cooperation, all of which are carried out by the internal student work line or ideological and political researchers in colleges and universities, and the distribution is also relatively scattered. There are few core authors, most of which are isolated points, and no real core author group in this field has been formed. With Qu Lili Li, Hou Yunchen, Zhang Jingke and Jiang Junyi as the core authors, several research teams are formed. However, their cooperation is often limited within the team, lacking of external communication and cooperation. As a result, the research in the field of precision funding in colleges and universities in China has not yet formed a relatively stable research group.

\subsection{There are too many repetitive studies and insufficient micro studies.}

There is too much repeated research, and the homogeneity of research is more common. In the research, it is found that there are more researches on students with financial difficulties, financial aid system and financial education, and even several articles on these topics were published in the same journal in the same year. Among them, the research enthusiasm for "students with family financial difficulties" has surpassed the high-frequency words such as funded education and targeted poverty alleviation, indicating that students with family financial difficulties have a very important position in the field of precision funding research in colleges and universities. Scientifically and accurately identify students with financial difficulties from families. "Current research involves more macro-level institutional mechanisms, model studies, and practical discussions, but there is less research in micro-fields such as the establishment of a model for identifying students with family financial difficulties. Even the existing micro-field research has emerged. Similar models, single content, superficial research and other issues." The results identified by students with financial difficulties from families are directly related to the flow of funding resources, the realization of education equity, and even the victory of the precise fight against poverty. It has important theoretical value and practical significance. When reviewing the literature, it is found that the current

ISSN: 0010-8189

(C) CONVERTER 2020 
theoretical research of scholars in this area is mainly focused on the composition of identification indicators, the selection of identification subjects, and the discussion of evaluation operation methods. Various technologies, models and methods used in the evaluation and identification of students from poor families are still in the primary stage, with simple method and single statistical method. How to build the accurate identification and evaluation model of college students with financial difficulties is one of the problems that need to be solved in order to better implement the accurate funding work. Therefore, it is urgent to change the current superficial research, which should be in line with the current reality, and adopt a variety of methods to carry out targeted research.

6.3 The research method is single, and the in-depth research is not enough.

In the existing research, most of the qualitative analysis method is used, mainly on the construction of the funding system, funding model, implementation path and so on, while the results of quantitative research are very limited "the concept of "precision" is based on quantification, "precision" mainly refers to simplification and easy operation, "precision" refers to quantification, refinement and operability [8]. To study the implementation path of "precision funding", we need to first study the method of "precision identification" and propose a variety of quantitative analysis and data modeling methods. However, at present, the quantitative research methods for financial aid and identification of college students with financial difficulties are relatively few and single, which are mainly limited to using analytic hierarchy process to determine the weight of factors and indicators, and establishing a hierarchical model for comprehensive evaluation of students' financial difficulties. The characteristic of this method is that the data is more intuitive, but there are many subjective judgment factors, and the indicators are often not scientifically tested and lack of persuasion; Due to the limitation of the design principle of the analytic hierarchy process, it is difficult to avoid human subjective factors and guarantee the reliability of the conclusion. It is worth noting that the current informatization construction of colleges and universities has entered a new era with the construction of "smart campus" as the theme. Students study and live in a common "smart campus". Their basic family information, arrears of loans, daily habits, campus consumption details, learning awards, scholarship evaluations and other information are recorded and digitized by various systems. These data truly, objectively and comprehensively reflect the behavior of students during school [9]. With the continuous increase of the upper-level application system of the "smart campus", the data accumulated in the campus information environment is also rapidly expanding, and a typical campus big data environment has been initially formed [10]. Adopt big data technology to restore students' real life state in the most objective and comprehensive way. Through big data analysis of student behavior trajectory, discover the interrelationships among students' diverse information and obtain more valuable guidance information, which can help the family economy The identification of students with difficulties provides quantitative indicators and targeted funding measures are adopted to verify and evaluate the funding system of colleges and universities, so as to realize the "precision" of college funding work.

6.4 There are many generalization researches, and quantitative research needs to be strengthened.

According to the current research situation, the use of speculative and qualitative research accounts for the majority, while the results of quantitative research are very limited [11], and the financial aid for students from families with financial difficulties in colleges and universities pays more attention to practice, if only at the speculative and qualitative level research, it will be difficult to in-depth research on the indicator system, process specifications, and funding effects of precision funding at the practical level [11]. At the same time, most of the existing researches are based on sampling research around a certain place or a certain university, and the survey is not extensive enough, which leads to the extreme limitation of the generalization of its conclusions, and the scientificity and universality are not enough. Due to the lack of scientific and systematic theoretical guidance, some of the defined methods and procedures in the policy documents are difficult to operate, subjective and arbitrary, leading to the empty and general phenomenon of theoretical research, and it is difficult to realize the transfer of valuable experience. The practice process is not rigorous enough, and the lack of objective basis for quantitative standards also directly affects the fairness and impartiality of the

ISSN: 0010-8189

(C) CONVERTER 2020 
funding work, affects the effect of the funding work, and arouses widespread concern about the unfair distribution of educational resources by the society and the government. Therefore, the precise realization of funding for students in need is a major issue in the current funding work for colleges and universities. Only in this way can we ensure the true realization of educational equity and ensure that we can win the battle against poverty.

\section{Acknowledgements}

This work was supported by the Research Projects of Philosophy and Social Sciences in Colleges and Universities of Jiangsu Province under Grant 2018SJSZ439, and in part by the Humanities and Social Sciences Foundation of the Ministry of Education under Grant 18YJCZH229, and in part by the 13th Five-Year Plan Project of Educational Science in Jiangsu Province under Grant X-a/2018/10.

\section{References}

[1] ZHANG Jian. Improve and Optimize the Financial Aid System for Students from Poor Families in Colleges and Universities. Social Sciences Review, 2012, (02):58-59.

[2] XUE Xiaoli, WU Yishan. Bibliometric Study on Interdisciplinary Research Articles and Its Visualization. Journal of Intelligence, 2014, (07):126-131.

[3] YANG Faping. The Evolutionary Context and Hot Spots of Research on China's Urban-rural Relationship (2000-2017)-Based on Citespace Bibliometric Analysis. Journal of Hubei Administration Institute, 2018, (04):7-7.

[4] CAI Wenbo, YANG Lixue. The Development of the Quality Education Research of China's University Students since the 21st Century. Review of Higher Education, 2018, (06):2-2.

[5] XIAO Guofang, LI Jianqiang. Research Topics on Technology Transfer and Knowledge Mapping Analysis based on SSCI. Library Journal, 2014, (05):82-87.

[6] JIN Jingyang. Identifying University Students in Poverty and Financing Problem Research. Hebei Normal University, 2013.

[7] LIN Shuxian. The Exploration of "Three Precision" Funding Model to Help the Students to Grow Up-Take Guangdong Vocational College of Agriculture, Industry and Commerce as An Example. Journal of Kaifeng Institute of Education, 2018, (09):3-3.

[8] YI Na; XU Jianjun; YAN Limei; HUANG Lin; Task optimization and scheduling of distributed cyber-physical system based on improved ant colony algorithm, Future Generation Computer Systems, 2020, 109:134-148. 3.

[9] Xu Jianjun, Wang Bao'e, Yan Limei, Li Zhanping. The Strategy of the Smart Home Energy Optimization Control of the Hybrid Energy Coordinated Control. Transactions of China Electrotechnical Society, 2017, 32(12): 214-223.

[10] Yankai Xu. Development of Ultrasonic Small Flow Measuring Instrument Based on STC89C52. 2019 2nd International Conference on Safety Produce Informatization, IEEE. Chongqing, Nov. 28-30, 2019, p:394-397

[11] Xu, J., Huang, L., Yin, S. et al. All-fiber self-mixing interferometer for displacement measurement based on the quadrature demodulation technique. Opt Rev. 2018, 25(1):40-45.

[12] SUN Yangbo. Research and Development of University Student Behavior Data Analysis System Based on Big Data Mining. North China Electric Power University, 2017.

[13] WANG Jiguang. A Meta-analysis of the Research on the Integration of Industry and Education in the Field of Higher Vocational Education. Journal of Vocational Education, 2017, (03):6-6. 